\title{
SEMISIMPLE ACTIONS OF MAPPING CLASS GROUPS ON CAT(0) SPACES
}

\author{
MARTIN R. BRIDSON
}

\begin{abstract}
Let $\Sigma$ be an orientable surface of finite type and let $\operatorname{Mod}(\Sigma)$ be its mapping class group. We consider actions of $\operatorname{Mod}(\Sigma)$ by semisimple isometries on complete $\mathrm{CAT}(0)$ spaces. If the genus of $\Sigma$ is at least 3, then in any such action all Dehn twists act as elliptic isometries. The action of $\operatorname{Mod}(\Sigma)$ on the completion of Teichmüller space with the Weil-Petersson metric shows that there are interesting actions of this type. Whenever the mapping class group of a closed orientable surface of genus $g$ acts by semisimple isometries on a complete $\mathrm{CAT}(0)$ space of dimension less than $g$ it must fix a point. The mapping class group of a closed surface of genus 2 acts properly by semisimple isometries on a complete CAT(0) space of dimension 18.
\end{abstract}

\section{INTRODUCTION}

This article concerns actions of mapping class groups by isometries on complete CAT(0) spaces. It records the contents of the lecture that I gave at Bill Harvey's 65th birthday conference at Anogia, Crete in July 2007.

A CAT(0) space is a geodesic metric space in which each geodesic triangle is no fatter than a triangle in the Euclidean plane that has the same edge lengths (see Definition 11). Classical examples include complete 1-connected Riemannian manifolds with non-positive sectional curvature and metric trees. The isometries of a CAT(0) space $X$ divide naturally into two classes: the semisimple isometries are those for which there exists $x_{0} \in X$ such that $d\left(\gamma \cdot x_{0}, x_{0}\right)=|\gamma|$ where $|\gamma|:=\inf \{d(\gamma . y, y) \mid y \in X\}$; the remaining isometries are said to be parabolic. Semisimple isometries are further divided into hyperbolics, for which $|\gamma|>0$, and elliptics, which have fixed points. Parabolics can be divided into neutral parabolics, for which $|\gamma|=0$, and non-neutral parabolics. If $X$ is a polyhedral space with only finitely many isometry types of cells, then all isometries of $X$ are semisimple [Bri 1999].

1991 Mathematics Subject Classification. 20F67, 57M50.

Key words and phrases. mapping class groups, $\mathrm{CAT}(0)$ spaces, dimension, fixed-point theorems.

This research was supported by a Senior Fellowship from the EPSRC and a Royal Society Wolfson Research Merit Award. To appear in "The Geometry of Riemann Surfaces", LMS Lecture Notes 368 . 
E. Cartan [Ca 1926] proved that the natural metric on a symmetric space of non-compact type has non-positive sectional curvature. This gives an action of the mapping class group on a complete $\mathrm{CAT}(0)$ space: the morphism $\operatorname{Mod}\left(\Sigma_{g}\right) \rightarrow \operatorname{Sp}(2 g, \mathbb{Z})$ induced by the action of $\operatorname{Mod}\left(\Sigma_{g}\right)$ on the first homology of $\Sigma_{g}$, the closed orientable surface of genus $g$, gives an action of $\operatorname{Mod}\left(\Sigma_{g}\right)$ by isometries on the symmetric space for the symplectic group $\operatorname{Sp}(2 g, \mathbb{R})$. In this action, the Dehn twists in non-separating curves act as neutral parabolics.

In the fruitful analogy between mapping class groups and lattices in semisimple Lie groups, Teichmüller space takes the role of the symmetric space. Unfortunately, the Teichmüller metric does not have non-positive curvature [Mas 1975]. On the other hand, the Weil-Petersson metric, although not complete, does have non-positive curvature [Wol 1975, Wol 1987]. Since the completion of a CAT(0) space is again a CAT(0) space [BriH 1999] p.187, it seems natural to complete the Weil-Petersson metric and to examine the action of the mapping class group on the completion $\overline{\mathcal{T}}$ in order to elucidate the structure of the group.

Theorem A. Let $\Sigma$ be an orientable surface of finite type with negative euler characteristic and empty boundary. The action of $\operatorname{Mod}(\Sigma)$ on the completion of Teichmüller space in the Weil-Petersson metric is by semisimple isometries. All Dehn twists act as elliptic isometries (i.e. have fixed points).

This theorem is a restatement of results in the literature but I wanted to highlight it as a motivating example. The essential points in the proof are described in Section 3. The fact that the Dehn twists have fixed points in the action on $\overline{\mathcal{T}}$ is a manifestation of a general phenomenon:

Theorem B. Let $\Sigma$ be an orientable surface of finite type with genus $g \geq 3$. Whenever $\operatorname{Mod}(\Sigma)$ acts by semisimple isometries on a complete $\mathrm{CAT}(0)$ space, all Dehn twists in $\operatorname{Mod}(\Sigma)$ act as elliptic isometries (i.e. have fixed points).

One proves this theorem by comparing the centralizers of Dehn twists in mapping class groups with the centralizers of hyperbolic elements in isometry groups of $\mathrm{CAT}(0)$ spaces - see Section 2, The action of $\operatorname{Mod}(\Sigma)$ on the symmetric space for $\operatorname{Sp}(2 g, \mathbb{R})$ shows that one must weaken the conclusion of Theorem $B$ if one wants to drop the hypothesis that $\operatorname{Mod}(\Sigma)$ is acting by semisimple isometries. The appropriate conclusion is that the Dehn twists act either as elliptics or as neutral parabolics (see Theorem 11).

Theorem B provides information about actions of finite-index subgroups of mapping class groups. For if $H$ is a subgroup of index $n$ acting by semisimple isometries on a $\operatorname{CAT}(0)$ space $X$, then the induced action of $\operatorname{Mod}(\Sigma)$ on $X^{n}$ is again by semisimple isometries: $\gamma \in \operatorname{Mod}(\Sigma)$ will be elliptic (resp. hyperbolic) in the induced action if and only if any power of $\gamma$ that lies in $H$ was elliptic (resp. hyperbolic) in the original action (cf. remark 1). 
The situation for genus 2 surfaces is quite different, as I shall explain in Section 6,

Theorem C. The mapping class group of a closed orientable surface of genus 2 acts properly by semisimple isometries on a complete $\mathrm{CAT}(0)$ space of dimension 18.

The properness of the action in Theorem $\mathrm{C}$ contrasts sharply with the nature of the actions in Theorems A, B and D.

I do not know if the mapping class group of a surface of genus $g>1$ can admit an action by semisimple isometries, without a global fixed point, on a complete $\mathrm{CAT}(0)$ space whose dimension is less than that of the Teichmüller space. However, one can give a lower bound on this dimension that is linear in $g$ (cf. Questions 13 and 14 of [BriV 2006] and Problem 6.1 in [Farb 2006]). To avoid complications, I shall state this only in the closed case.

Theorem D. Whenever the mapping class group of a closed orientable surface of genus $g$ acts by semisimple isometries on a complete CAT(0) space of dimension less than $g$ it fixes a point.

Here, "dimension" means topological covering dimension. An outline of the proof is given in Section 5; the details are given in [Bri2]. The strategy of proof is based on the "ample duplication" criterion in [Bri1]. The semisimple hypothesis can be weakened: it is sufficient to assume that there are no nonneutral parabolics, cf. Theorem 1 .

I make no claim regarding the sharpness of the dimension bounds in Theorems $\mathrm{C}$ and $\mathrm{D}$.

The lecture on which this paper is based was a refinement of a lecture that I gave on 8 December 2000 in Bill Harvey's seminar at King's College London. Bill worked tirelessly over many years to maintain a geometry and topology seminar in London. Throughout that time he shared many insights with visiting researchers and always entertained them generously. His mathematical writings display the same generosity of spirit: he has written clearly and openly about his ideas rather than hoarding them until some arcane goal was achieved. The benefits of this openness are most clear in his highly prescient and influential papers introducing the curve complex [Harv 1981, Harv 1979].

The book [Harv 1977] on discrete groups and automorphic forms that Bill edited in 1977 had a great influence on me when I was a graduate study. At a more personal level, he and his wife Michele have been immensely kind to me and my family over many years. It is therefore with the greatest pleasure that I dedicate these observations about the mapping class group to him on the occasion of his sixty fifth birthday. 


\section{Centralizers, fixed points And Theorem B}

Definition 1. Let $X$ be a geodesic metric space. A geodesic triangle $\Delta$ in $X$ consists of three points $a, b, c \in X$ and three geodesics $[a, b],[b, c],[c, a]$. Let $\bar{\Delta} \subset \mathbb{E}^{2}$ be a triangle in the Euclidean plane with the same edge lengths as $\Delta$ and let $\bar{x} \mapsto x$ denote the map $\bar{\Delta} \rightarrow \Delta$ that sends each side of $\bar{\Delta}$ isometrically onto the corresponding side of $\Delta$. One says that $X$ is a $\mathrm{CAT}(0)$ space if for all $\Delta$ and all $\bar{x}, \bar{y} \in \bar{\Delta}$ the inequality $d_{X}(x, y) \leq d_{\mathbb{E}^{2}}(\bar{x}, \bar{y})$ holds.

Note that in a $\mathrm{CAT}(0)$ space there is a unique geodesic $[x, y]$ joining each pair of points $x, y \in X$. A subspace $Y \subset X$ is said to be convex if $\left[y, y^{\prime}\right] \subset Y$ whenever $y, y^{\prime} \in Y$.

Lemma 1. If $X$ is a complete CAT(0) space then an isometry $\gamma$ of $X$ is hyperbolic if and only if $|\gamma|>0$ and there is a $\gamma$-invariant convex subspace of $X$ isometric to $\mathbb{R}$. (Each such subspace is called an axis for $\gamma$.)

Proposition 1. Let $\Gamma$ be a group acting by isometries on a complete $\mathrm{CAT}(0)$ space $X$. If $\gamma \in \Gamma$ acts as a hyperbolic isometry then $\gamma$ has infinite order in the abelianisation of its centralizer $Z_{\Gamma}(\gamma)$.

Proof. This is proved on page 234 of BriH 1999] (remark 6.13). The main points are these: if $\gamma$ is hyperbolic then the union of the axes of $\gamma$ splits isometrically as $Y \times \mathbb{R}$; this subspace and its splitting are preserved by $Z_{\Gamma}(\gamma)$; the action on the second factor gives a homomorphism from $Z_{\Gamma}(\gamma)$ to the abelian group $\operatorname{Isom}_{+}(\mathbb{R})$, in which the image of $\gamma$ is non-trivial.

In the light of this proposition, in order to prove Theorem B it suffices to show that if $\Sigma$ is a surface of finite type with genus $g \geq 3$, then the Dehn twist $T$ about any simple closed curve $c$ in $\Sigma$ does not have infinite order in the abelianisation of its centralizer.

Proposition 2. If $\Sigma$ is an orientable surface of finite type that has genus at least 3 (with any number of boundary components and punctures) and if $T$ is the Dehn twist about any simple closed curve $c$ in $\Sigma$, then the abelianisation of the centralizer of $T$ in $\operatorname{Mod}(\Sigma)$ is finite.

Proof. The centralizer of $T$ in $\operatorname{Mod}(\Sigma)$ consists of mapping classes of homeomorphisms that leave $c$ invariant. This is a homomorphic image of the mapping class group of the surface obtained by cutting $\Sigma$ along $c$. (This surface has two boundary components corresponding to $c$ and hence two Dehn twists mapping to $T$.) Since $\Sigma$ has genus at least 3, at least one component of the cut-open surface has genus $g \geq 2$. The mapping class group of such a surface has finite abelianisation - see [Kor 2002] for a concise survey and references.

Remark 1. As we remarked in the introduction, Theorem $B$ gives restrictions on how subgroups of finite index in $\operatorname{Mod}(\Sigma)$ can act on $\mathrm{CAT}(0)$ spaces. For example, given an orientable surface of genus $g \geq 3$ and a homomorphism $\phi$ 
from a subgroup $H<\operatorname{Mod}(\Sigma)$ of index $n$ to a group $G$ that acts by hyperbolic isometries on a complete $\mathrm{CAT}(0)$ space $X$, we can apply Theorem $B$ to the induced action 1 of $\operatorname{Mod}(\Sigma)$ on $X^{n}$ and hence deduce that any power of a Dehn twist that lies in $H$ must lie in the kernel of $\phi$. Taking $G=\mathbb{Z}$ tells us that powers of Dehn twists cannot have infinite image in the abelianisation of $H$. A more explicit proof of this last fact was given recently by Andew Putman [Put].

I am grateful to Pierre-Emmanuel Caprace, Dawid Kielak, Anders Karlsson and Nicolas Monod for their comments concerning the following extension of Theorem B.

Theorem 1. Let $\Sigma$ be an orientable surface of finite type with genus $g \geq 3$. Whenever $\operatorname{Mod}(\Sigma)$ acts by isometries on a complete $\mathrm{CAT}(0)$ space, each Dehn twist $T \in \operatorname{Mod}(\Sigma)$ acts either as an elliptic isometry or as a neutral parabolic (i.e. $|T|=0$ ).

Proof. The proof of Theorem $\mathrm{B}$ will apply provided we can extend Proposition 1 to cover non-neutral parabolics. In order to appreciate this extension, the reader should be familiar with the basic theory of Busemann functions in CAT(0) spaces, BriH 1999] Chap. II.8.

If $\gamma$ is a parabolic isometry with $|\gamma|>0$, then a special case of a result of Karlsson and Margulis [KaMa 1999] (cf. [Ka 2002], p. 285) shows that $\gamma$ has a unique fixed point at infinity $\xi \in \partial X$ with the property that $\frac{1}{n} d\left(\gamma^{n} . x, c(n|\gamma|)\right) \rightarrow$ 0 as $n \rightarrow \infty$ for every $x \in X$ and every geodesic ray $c:[0, \infty) \rightarrow X$ with $c(\infty)=\xi$. Now, $Z_{\Gamma}(\gamma)$ fixes $\xi$ and acts on any Busemann function centred at $\xi$ by the formula $z \cdot \beta(t)=\beta(t)+\phi(z)$, where $\phi: Z_{\Gamma}(\gamma) \rightarrow \mathbb{R}$ is a homomorphism. Since $\phi(\gamma)=-|\gamma|$, this is only possible if $\gamma$ has infinite order in the abelianisation of $Z_{\Gamma}(\gamma)$.

I. Kapovich and B. Leeb [KL 1995] were the first to prove that if $g \geq 3$ then $\operatorname{Mod}\left(\Sigma_{g}\right)$ cannot act properly by semisimple isometries on a complete $\operatorname{CAT}(0)$ space; cf. [BriH 1999] p. 257.

\section{Augmented Teichmüller space And Theorem A}

Let $\Sigma$ be an orientable hyperbolic surface of finite type with empty boundary and let $\overline{\mathcal{T}}$ denote the completion of its Teichmüller space equipped with the Weil-Petersson metric. $\overline{\mathcal{T}}$ is equivariantly homeomorphic to the augmented Teichmüller space defined by Abikoff Abi 1977. Wolpert's concise survey Wol 2006] provides a clear introduction and ample references to the facts that we need here.

\footnotetext{
${ }^{1}$ One can regard this as "multiplicative induction" in the sense of tomD 1987] p. 35: if $H<G$ has index $n$ and acts on $X$ then one identifies $X^{n}$ with the space of $H$-equivariant maps $f: G \rightarrow X$ and considers the (right) action $(g . f)(\gamma):=f(\gamma g)$; a power of each $g \in G$ preserves the factors of $X^{n}$, so it follows from [BriH 1999] p. 231-232 that the action of $G$ is by semisimple isometries if the action of $H$ is.
} 
Masur [Mas 1976] describes the metric structure of $\overline{\mathcal{T}} \backslash \mathcal{T}$ as follows. It is a union of strata $\mathcal{T}_{C}$ corresponding to the homotopy classes of systems $C$ of disjoint, non-parallel, simple closed curves on $\Sigma$. The stratum corresponding to $C$ is the Teichmüller space of the nodal surface obtained by shrinking each loop $c \in C$ to a pair of cusps (punctures); more explicitly, it is the product of Teichmüller spaces for the components of $\Sigma \backslash \bigcup C$ (with punctures in place of the pinched curves), each equipped with its Weil-Petersson metric. The identification of $\mathcal{T}_{C}$ with this product of Teichmüller spaces is equivariant with respect to the natural map from the subgroup of $\operatorname{Mod}(\Sigma)$ that preserves $C$ and its components to the mapping class group of the nodal surface. Importantly, $\mathcal{T}_{C} \subset \overline{\mathcal{T}}$ is a convex subspace [DaWe 2003].

The Dehn twists (and hence multi-twists $\mu$ ) in the curves of $C$ act trivially on $\mathcal{T}_{C}$ and hence are elliptic isometries of $\overline{\mathcal{T}}$.

Daskalopoulos and Wentworth [DaWe 2003] proved that every pseudo-Anosov element $\psi$ of $\operatorname{Mod}(\Sigma)$ acts as a hyperbolic isometry of $\overline{\mathcal{T}}$; indeed each has an axis contained in $\mathcal{T}$. If $\rho \in \operatorname{Mod}(\Sigma)$ leaves invariant a curve system $C$ and each of the components of $\Sigma \backslash C$, then either it is a multi-twist (and hence acts as an elliptic isometry of $\overline{\mathcal{T}}$ ) or else it acts as a pseudo-Anosov on one of the components of $\Sigma \backslash C$. In the latter case $\rho$ will act as a hyperbolic isometry of $\mathcal{T}_{C}$, and hence of $\overline{\mathcal{T}}$, since $\mathcal{T}_{C} \subset \overline{\mathcal{T}}$ is convex. An isometry of a complete $\mathrm{CAT}(0)$ space is hyperbolic (resp. elliptic) if and only if every proper power of it is hyperbolic (resp. elliptic) [BriH 1999], p. 231-232. Every element of the mapping class group has a proper power that is one of the three types $\mu, \psi, \rho$ considered above [Thu 1988]. Thus Theorem A is proved.

Remarks 2. (1) I want to emphasize once again that I stated Theorem A only to provide context: nothing in the proof is original. Moreover, since $I$ first wrote this note, Ursula Hamenstadt has given essentially the same proof in $\underline{\mathrm{Ham}}$.

(2) Masur-Wolf [MasW 2002] and Brock-Margalit BroM 2007 have shown that $\operatorname{Mod}(\Sigma)$ is the full isometry group of $\overline{\mathcal{T}}$. I am grateful to Jeff Brock for a helpful correspondence on this point.

(3) In the proof of Theorem A, the roots of multitwists emerged as the only elliptic isometries (provided that we regard the identity as a multitwist). Combining this observation with Theorem $B$, we see that any homomorphism from the mapping class group of a surface of genus $g \geq 3$ to another mapping class group must send roots of multitwists to roots of multitwists. This contrasts with the fact that there are injective homomorphisms between mapping class groups of once-punctured surfaces of higher genus that send pseudo-Anosov elements to multitwists [ALS]. 


\section{CRITERIA FOR COMMON FIXED POINTS}

The classical theorem of Helly concerns the combinatorics of families of convex subsets in $\mathbb{R}^{n}$. There are many variations on this theorem in the literature. For our purposes the following will be sufficient (see [Bri1], [Farb] and references therein).

Proposition 3. Let $X$ be a complete CAT(0) space of (topological covering) dimension at most $n$, and let $C_{1}, \ldots, C_{N} \subset X$ be closed convex subsets. If every $(n+1)$ of the $C_{i}$ have a point of intersection, then $\bigcap_{i=1}^{N} C_{i} \neq \emptyset$.

When applied to the fixed point sets $C_{i}=\operatorname{Fix}\left(s_{i}\right)$ with $s_{i} \in S$, this implies:

Corollary 1. Let $\Gamma$ be a group acting by isometries on a complete $\mathrm{CAT}(0)$ space $X$ of dimension at most $n$ and suppose that $\Gamma$ is generated by the finite set $S$. If every $(n+1)$-element subset of $S$ fixes a point of $X$, then $\Gamma$ has a fixed point in $X$.

We shall need a refinement of this result that relies on the following wellknown proposition, BriH 1999] p.179. We write $\operatorname{Isom}(X)$ for the group of isometries of a metric space $X$ and $\operatorname{Ball}_{r}(x)$ for the closed ball of radius $r>0$ about $x \in X$. Given a subspace $Y \subseteq X$, let $r_{Y}:=\inf \{r \mid Y \subseteq$ $\operatorname{Ball}_{r}(x)$, some $\left.x \in X\right\}$.

Proposition 4. If $X$ is a complete CAT(0) space and $Y$ is a bounded subset, then there is a unique point $c_{Y} \in X$ such that $Y \subseteq \operatorname{Ball}_{r}\left(c_{Y}\right)$.

Corollary 2. Let $X$ be a complete $\mathrm{CAT}(0)$ space. If $H<\operatorname{Isom}(X)$ has a bounded orbit then $H$ has a fixed point.

Proof. The centre $c_{O}$ of any $H$-orbit $O$ will be a fixed point.

Corollary 3. Let $X$ be a complete CAT(0) space. If the groups $H_{1}, \ldots, H_{\ell}<$ $\operatorname{Isom}(X)$ commute and $\operatorname{Fix}\left(H_{i}\right)$ is non-empty for $i=1, \ldots, \ell$, then $\bigcap_{i=1}^{\ell} \operatorname{Fix}\left(H_{i}\right)$ is non-empty.

Proof. A simple induction reduces us to the case $\ell=2$. Since $\operatorname{Fix}\left(H_{2}\right)$ is non-empty, each $H_{2}$-orbit is bounded. As $H_{1}$ and $H_{2} \operatorname{commute}, \operatorname{Fix}\left(H_{1}\right)$ is $H_{2}$-invariant and therefore contains an $H_{2}$-orbit. As $\operatorname{Fix}\left(H_{1}\right)$ is convex, the centre of this (bounded) orbit is also in $\operatorname{Fix}\left(H_{1}\right)$, and therefore is fixed by $H_{1} \cup H_{2}$.

Building on these elementary observations, one can prove the following; see Bri1.

Proposition 5 (Bootstrap Lemma). Let $k_{1}, \ldots, k_{n}$ be positive integers and let $X$ be a complete CAT(0) space of dimension less than $k_{1}+\cdots+k_{n}$. Let $S_{1}, \ldots, S_{n} \subset \operatorname{Isom}(X)$ be subsets with $\left[s_{i}, s_{j}\right]=1$ for all $s_{i} \in S_{i}$ and $s_{j} \in$ $S_{j}(i \neq j)$. 
If, for $i=1, \ldots, n$, each $k_{i}$-element subset of $S_{i}$ has a fixed point in $X$, then for some $i$ every finite subset of $S_{i}$ has a fixed point.

When applying the Bootstrap Lemma one has to overcome the fact that the conclusion only applies to some $S_{i}$. A convenient way of gaining more control is to restrict attention to conjugate sets.

Corollary 4 (Conjugate Bootstrap). Let $k$ and $n$ be positive integers and let $X$ be a complete CAT(0) space of dimension less than $n k$. Let $S_{1}, \ldots, S_{n}$ be conjugates of a subset $S \subset \operatorname{Isom}(X)$ with $\left[s_{i}, s_{j}\right]=1$ for all $s_{i} \in S_{i}$ and $s_{j} \in S_{j}(i \neq j)$.

If each $k$-element subset of $S$ has a fixed point in $X$, then so does each finite subset of $S$.

\section{Some SuRfaCe TOPOLOGY}

The reader will recall that, given a closed orientable surface $\Sigma$ and two compact homeomorphic sub-surfaces with boundary $T, T^{\prime} \subset \Sigma$, there exists an automorphism of $\Sigma$ taking $T$ to $T^{\prime}$ if and only $\Sigma \backslash T$ and $\Sigma \backslash T^{\prime}$ are homeomorphic. In particular, two homeomorphic sub-surfaces are in the same orbit under the action of $\operatorname{Homeo}(\Sigma)$ if the complement of each is connected.

The relevance of this observation to our purposes is explained by the following lemma, which will be used in tandem with the Conjugate Bootstrap.

Lemma 2. Let $H$ be the subgroup of $\operatorname{Mod}(\Sigma)$ generated by the Dehn twists in a set $C$ of loops all of which are contained in a compact sub-surface $T \subset \Sigma$ with connected complement. If $\Sigma$ contains $m$ mutually disjoint sub-surfaces $T_{i}$ homeomorphic to $T$, each with connected complement, then $\operatorname{Mod}(\Sigma)$ contains $m$ mutually-commuting conjugates $H_{i}$ of $H$.

Proof. Since the complement of each $T_{i}$ is connected, there is a homeomorphism $\phi_{i}$ of $\Sigma$ carrying $T$ to $T_{i}$. Define $H_{i}$ to be the subgroup of $\operatorname{Mod}(\Sigma)$ generated by the Dehn twists in the loops $\phi_{i}(C)$. Since the various $H_{i}$ are supported in disjoint sub-surfaces, they commute.

5.1. The Lickorish generators. Raymond Lickorish [Lic 1964] proved that the mapping class group of a closed orientable surface of genus $g$ is generated by the Dehn twists in $3 g-1$ non-separating loops, each pair of which intersects in at most one point. Let Lick denote this set of loops.

We say that a subset $S \subset$ Lick is connected if the union $U(S)$ of the loops in $S$ is connected. An analysis of Lick reveals the following fact, whose proof is deferred to Bri2]. In this statement all sub-surfaces are assumed to be compact.

Proposition 6. Let $S \subset$ Lick be a connected subset. 
(1) If $|S|=2 \ell$ is even, then $U(S)$ is either contained in a sub-surface of genus $\ell$ with 1 boundary component, or else in a non-separating subsurface of genus at most $\ell-1$ with 3 boundary components.

(2) If $|S|=2 \ell+1$ is odd, then $U(S)$ is either contained in a non-separating subsurface of genus $\ell$ with at most 2 boundary components, or else in a non-separating sub-surface of genus at most $(\ell-1)$ that has at most 3 boundary components.

5.2. The Proof of Theorem D: an outline. We must argue that when the mapping class group of a closed orientable surface of genus $g$ acts without neutral parabolics on a complete $\mathrm{CAT}(0)$ space $X$ of dimension less than $g$ it must fix a point.

The case $g=1$ is trivial. A complete CAT(0) space of dimension 1 is an $\mathbb{R}$-tree, so for $g=2$ the assertion of the theorem is that the mapping class group of a genus 2 surface has property FR. This was proved by Culler and Vogtmann $\mathrm{CuVo} 1996$.

Assume $g \geq 3$. According to Corollary 1, we will be done if we can show that each subset $S \subset$ Lick with $|S| \leq g$ has a fixed point in $X$. We proceed by induction on $|S|$. Theorem 1 covers the base case $|S|=1$.

If $S$ is not connected, say $S=S_{1} \cup S_{2}$ with $U\left(S_{1}\right) \cap U\left(S_{2}\right)=\emptyset$, then the subgroups $\left\langle S_{1}\right\rangle$ and $\left\langle S_{2}\right\rangle$ commute. Each has a fixed point since $\left|S_{i}\right|<|S|$, so Corollary 3 tells us that $S$ has a fixed point.

Suppose now that $S$ is connected. If $|S|=2 \ell$ is even then Proposition 6] tells us that $U(S)$ is contained either in a sub-surface of genus $\ell$ with 1 boundary component or else in a non-separating sub-surface of genus $\ell-1$ with 3 boundary components. In either case one can fit $\lfloor g / \ell\rfloor$ disjoint copies of this sub-surface into $\Sigma_{g}$. Lemma 2 then provides us with $\lfloor g / \ell\rfloor$ mutuallycommuting conjugates of $\langle S\rangle$. As all proper subsets of $S$ are assumed to have a fixed point and the dimension of $X$ is less than $(2 \ell-1)\lfloor g / \ell\rfloor$, the Conjugate Bootstrap (Corollary 4) tells us that $S$ will have a fixed point.

The argument for $|S|$ odd is similar. For a detailed proof, see [Bri2].

\section{A proper semisimple aCtion of $\operatorname{Mod}\left(\Sigma_{2}\right)$}

Theorem 2. The mapping class group of a closed surface of genus 2 acts properly by semisimple isometries on a complete $\mathrm{CAT}(0)$ space of dimension 18.

Proof. The hyperelliptic involution $\tau$ is central in $\operatorname{Mod}\left(\Sigma_{2}\right)$. The quotient orbifold $\Sigma_{2} /\langle\tau\rangle$ is a sphere with 6 marked points and the action of $\operatorname{Mod}\left(\Sigma_{2}\right)$ on this quotient induces a homomorphism $\operatorname{Mod}\left(\Sigma_{2}\right) \rightarrow \operatorname{Mod}\left(\Sigma_{0,6}\right)$. This is onto [BiHi 1971] and the kernel is $\langle\tau\rangle$. Thus we have a short exact sequence

$$
1 \rightarrow \mathbb{Z}_{2} \rightarrow \operatorname{Mod}\left(\Sigma_{2}\right) \rightarrow \operatorname{Mod}\left(\Sigma_{0,6}\right) \rightarrow 1
$$


For each positive integer $n \geq 2$ there is a natural homomorphism from the braid group $B_{n}$ to $\operatorname{Mod}\left(\Sigma_{0, n+1}\right)$; the image of this map is of index $(n+1)$ and the kernel is the centre of $B_{n}$, which is infinite cyclic. This map admits the following geometric interpretation. Regard $B_{n}$ as the mapping class group of the $n$ punctured disc $D$ with 1 boundary component. One maps $B_{n}$ to $\operatorname{Mod}\left(\Sigma_{0, n+1}\right)$ by attaching the boundary of a once-punctured disc to $\partial D$ and extending homeomorphisms of $D$ by the identity on the attached disc. The image of $B_{n}$ is the subgroup of $\operatorname{Mod}\left(\Sigma_{0, n+1}\right)$ that stabilizes the puncture in the added disc; this has index $n+1$. The centre of $B_{n}$ is the mapping class of the Dehn twist $\zeta$ in a loop parallel to the boundary $\partial D$. This twist becomes trivial in $\operatorname{Mod}\left(\Sigma_{0, n+1}\right)$, and it generates the kernel of $B_{n} \rightarrow \operatorname{Mod}\left(\Sigma_{0, n+1}\right)$. Thus we have a second short exact sequence

$$
1 \rightarrow \mathbb{Z} \rightarrow B_{n} \rightarrow \Gamma \rightarrow 1
$$

where $\Gamma \subset \operatorname{Mod}\left(\Sigma_{0, n+1}\right)$ is of index $n+1$.

Brady and McCammond [BrMc] and independently Krammer (unpublished), showed that $B_{5}$ is the fundamental group of a compact non-positively curved piecewise-Euclidean complex $X$ of dimension 4 that has no free faces. It follows from BriH 1999] II.6.15(1) and II.6.16 that the universal cover of $X$ splits isometrically as a product $Y \times \mathbb{R}$ and that the quotient of $B_{5}$ by its centre acts properly on $Y$ (II.6.10(4) loc. cit.) by semisimple isometries (II.6.9 loc. cit.). By inducing, as in remark 1, we obtain an action of $\operatorname{Mod}\left(\Sigma_{0,6}\right)$ on $Y^{6}$ that is again proper and semisimple. And since the kernel of $\operatorname{Mod}\left(\Sigma_{2}\right) \rightarrow \operatorname{Mod}\left(\Sigma_{0,6}\right)$ is finite, the resulting action of $\operatorname{Mod}\left(\Sigma_{2}\right)$ on $Y^{6}$ is also proper.

\section{REFERENCES}

[Abi 1977] W. Abikoff, Degenerating families of Riemann surfaces, Ann. of Math. 105 (1977), 29-44.

[ALS] J. Aramayona, C.J. Leininger and J. Souto, Injections of mapping class groups, preprint, arXiv:0811.0841,

[BiHi 1971] J.S. Birman and H.M. Hilden, On the mapping class groups of closed surfaces as covering spaces, in "Advances in the theory of Riemann surfaces" (Stony Brook 1969), pp. 81-115, Ann. of Math. Studies 66, Princeton Univ. Press, Princeton NJ, 1971.

[BrMc] T. Brady and J. McCammond, A CAT(0) structure for the 5-string braid group, in preparation.

[Bri 1999] M.R. Bridson, On the semisimplicity of polyhedral isometries, Proc. Amer. Math. Soc. 127 (1999), 2143-2146.

[Bri1] M.R. Bridson, Helly's theorem, CAT(0) spaces, and actions of automorphism groups of free groups, preprint, Oxford, 2007.

[Bri2] M.R. Bridson, On the dimension of CAT(0) spaces where mapping class groups act, preprint, Oxford, 2009.

[BriH 1999] M.R. Bridson and A. Haefliger, "Metric spaces of nonpositive curvature", Grundlehren der Math. Wiss. 319, Springer-Verlag, Berlin, 1999. 
[BriV 2006] M.R. Bridson and K. Vogtmann, Automorphism groups of free groups, surface groups and free abelian groups, in "Problems on mapping class groups and related topics" (B. Farb, ed.), pp. 301-316, Proc. Sympos. Pure Math., 74, Amer. Math. Soc., Providence, RI, 2006.

[BroM 2007] J. Brock and D. Margalit, Weil-Petersson isometries via the pants complex, Proc. Amer. Math. Soc. 135 (2007), 795-803.

[Ca 1926] E. Cartan, Sur une classe remarquable d'espaces de Riemann, Bull. Soc. Math. France 54 (1926), 214-264.

[CuVo 1996] M. Culler and K. Vogtmann, A group theoretic criterion for property FA, Proc. Amer. Math. Soc. 124 (1996), 677-683.

[DaWe 2003] G. Daskalopoulos and R. Wentworth, Classification of Weil-Petersson isometries, Amer. J. Math. 125 (2003), 941-975.

[tomD 1987] T. tom Dieck, "Transformation groups", Studies in Mathematics 8, de Gruyter, Berlin-New York, 1987.

[Farb 2006] B. Farb, Some problems on mapping class groups and moduli space, in "Problems on mapping class groups and related topics" (B. Farb, ed.), pp. 11-55, Proc. Sympos. Pure Math., 74, Amer. Math. Soc., Providence, RI, 2006.

[Farb] B. Farb, Group actions and Helly's theorem, preprint, arXiv:0806.1692.

[Ham] U. Hamenstadt, Dynamical properties of the Weil-Petersson metric, preprint, arXiv:0901.4301.

[Harv 1977] W.J. Harvey (ed.), "Discrete groups and automorphic forms", Academic Press, London, 1977.

[Harv 1979] W.J. Harvey, Geometric structure of surface mapping class groups, in "Homological group Theory" (Durham 1977), pp. 255-269. London Math. Soc. Lecture Note Ser. 36, Cambridge Univ. Press, Cambridge-New York, 1979.

[Harv 1981] W.J. Harvey, Boundary structure of the modular group, in "Riemann surfaces and related topics" (Stony Brook 1978), pp. 245-251, Ann. of Math. Stud., 97, Princeton Univ. Press, Princeton NJ, 1981.

[Iv 2002] N.V. Ivanov, Mapping class groups, in "Handbook of geometric topology" (R. Daverman, R.B. Sher, eds.), Elsevier Science, Amsterdam, 2002, pp. 523633.

[KL 1995] M. Kapovich and B. Leeb, Actions of discrete groups on nonpositively curved spaces, Math. Ann. 306 (1996), no. 2, 341-352.

[Ka 2002] A. Karlsson, Nonexpanding maps, Busemann functions, and multiplicative ergodic theory, in "Rigidity in dynamics and geometry" (Cambridge, 2000), pp. 283-294. Springer-Verlag, Berlin, 2002.

[KaMa 1999] A. Karlsson and G. Margulis, A multiplicative ergodic theorem and nonpositively curved spaces, Comm. Math. Phys. 208 (1999), 107-123.

[Kor 2002] M. Korkmaz, Low-dimensional homology groups of mapping class groups: a survey, Turk. J. Math 26 (2002), 101-114.

[Lic 1964] W.B.R. Lickorish, A finite set of generators for the homeotopy group of a 2manifold, Proc. Cambridge Philos. Soc. 60 (1964), 769778.

[Lin 1971] M.R. Linch, On metrics in Teichmüller spaces, Ph.D. Thesis, Columbia Univ., New York, 1971.

[Mas 1975] H. Masur, On a class of geodesics in Teichmüller space, Ann. of Math. (2) 102 (1975), 205-221.

[Mas 1976] H. Masur, The extension of the Weil-Petersson metric to the boundary of Teichmüller space, Duke Math J. 43 (1976), 623-635. 
[MasW 2002] H. Masur and M. Wolf, The Weil-Petersson isometry group, Geom. Dedicata 93 (2002), 177-190.

[Put] A. Putman, A note on the abelianizations of finite-index subgroups of the mapping class group, preprint arXiv:0812.0017.

[Thu 1988] W.P. Thurston, On the geometry and dynamics of diffeomorphisms of surfaces, Bull. Amer. Math. Soc. 19 (1988), 417-431.

[Wol 1975] S.A. Wolpert, Noncompleteness of the Weil-Petersson metric for Teichmüller space, Pacific J. Math., 61 (1975), 573-577.

[Wol 1987] S.A. Wolpert, Geodesic length functions and the Nielsen problem, J. Diff. Geom. 25 (1987), 275-296.

[Wol 2006] S.A. Wolpert, Weil-Petersson perspectives, in "Problems on mapping class groups and related topics" (B. Farb, ed.), pp. 301-316, Proc. Sympos. Pure Math., 74, Amer. Math. Soc., Providence, RI, 2006.

Mathematical Institute, 24-29 St Giles', Oxford OX1 3LB, U.K.

E-mail address: bridson@maths.ox.ac.uk 